\title{
Effect of Waveform Parameters on Pulsed Glow Discharge in Air
}

\author{
Fengbo Tao ${ }^{1}$, Zhicheng Zhou ${ }^{1}$, Yong $\mathrm{Ma}^{1}$, Qiaogen Zhang ${ }^{2}$ \\ ${ }^{1}$ Jiangsu Electric Power Research Institute, Nanjing, China \\ ${ }^{2}$ School of Electrical Engineering, Xi'an Jiaotong University, Xi'an, China \\ Email: hvtaofb@gmail.com
}

Received August 25, 2012; revised September 26, 2012; accepted October 4, 2012

\begin{abstract}
The nanosecond single pulse was employed here to generate a large volume glow discharge between the wire-plane electrodes in air. In order to find requirements on pulse waveform for generation of a large volume discharge at atmospheric pressure, the effect of pulse risetime, pulse width, and amplitude on glow discharge has been widely investigated in this paper. Results reveal that a large volume glow discharge can be generated in an inhomogeneous electric field under the single pulse with the faster risetime, the lower peak amplitude. The pulse width has almost no influence on the density of glow discharge, but which has a great influence on the transition from glow discharge to streamer discharge. A model of inter-shielding-effect has been proposed to explain the influence of waveform parameters on pulsed glow discharge.
\end{abstract}

Keywords: Pulsed Discharge; Large Volume Glow Discharge; Inter-Shielding-Effect

\section{Introduction}

Gas plasma generated by pulse discharge is widely used in ozone generation [1-3], detoxification of gaseous pollution [4-6], material surface treatment [7,8], synthesis of nanostructured material $[9,10]$, etc. In order to improve the efficiency of the applications mentioned above, the homogeneous, large volume discharge is required. From the previous works, it can be seen that nanosecond pulse discharge in non-uniform electric field constructed by needles-plane or wire-plane electrodes is an efficient way to obtain large volume discharge. For instance, Ryo Ono and Tetsuji Oda have used the pulsed discharge between needles-plane electrodes to generate ozone and measured the ozone distribution in the discharge gap. R. A. Roush and R. K. Hutcherson focused on the exhaust gas detoxification efficiency by large volume discharge under different pulse risetime and pulse width [11-13]. Although the large volume discharge is widely used in the industry applications, the effect of waveform parameters on the formation of large volume discharge is not very clear yet. In order to find requirements on pulse waveform for generation of a large volume discharge at atmospheric pressure and understand more clearly the mechanism of pulsed discharge, a single pulse voltage with adjustable risetime, pulse width, amplitude etc is employed here to investigate the formation of the large volume discharge between the wire-plane electrodes with non-uniform electric field.

\section{Experimental Set-Up}

Figure 1 shows the schematic experimental circuit employed in this research. A single pulse with the pulse risetime from $10 \mathrm{~ns}$ to $500 \mathrm{~ns}$, pulse width from $100 \mathrm{~ns}$ to $2 \mu$ s, peak amplitude from $10 \mathrm{kV}$ to $50 \mathrm{kV}$ was applied on the wire-plane electrodes. A rogowski brass plane of $60 \mathrm{~mm}$ in diameter was used as the cathode, and a brass wire of $0.1 \mathrm{~mm}$ in diameter was used as the anode. Also, the wire of $100 \mathrm{~mm}$ in length, longer than the diameter of the cathode, was used to prevent the edge effect of the two electrodes. The gap distance was fixed at $8 \mathrm{~mm}$. Both electrodes were sealed in a polymethyl methacrylate container. The air pressure in the chamber can be adjusted in the range of $2 \mathrm{kPa}$ to $0.2 \mathrm{MPa}$.

The pulse voltage was measured with the voltage divider consisting of $\left(R_{1} \& C_{1}\right)$ and $\left(R_{2} \& C_{2}\right)$, which has the response time less than $5 \mathrm{~ns}$. The discharge current was measured with a current transducer (rogowski coil), which has the response time less than 5 ns. The voltage and current signals were recorded by a digitizing oscilloscope (Tektronix DPO4054) with a bandwidth of 500 $\mathrm{MHz}$. Considering that the discharge images are in correspondence with one-off discharge process caused by the nanosecond single pulse, there will be no superposition of repetitious discharges, so the exposure time is 


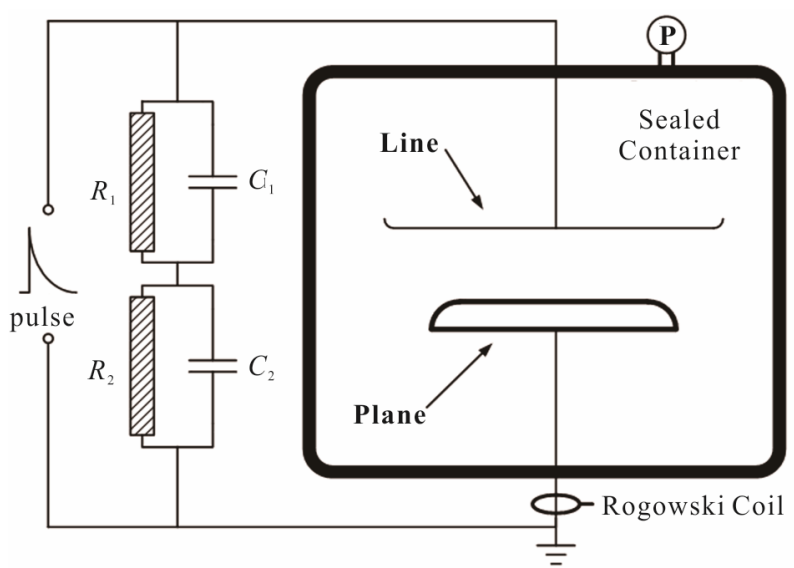

Figure 1. Schematic diagram of the electrode system and the experimental set-up.

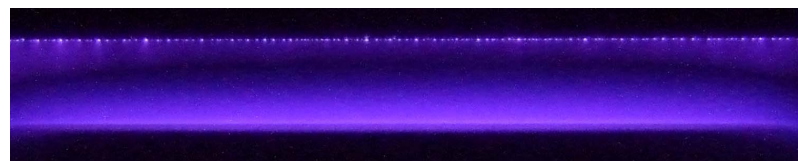

(a1)

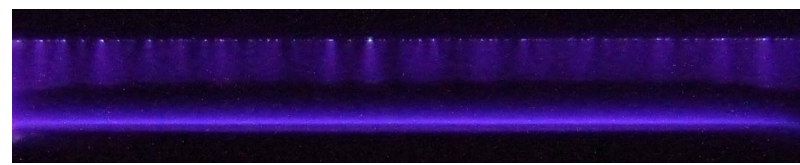

(a2)

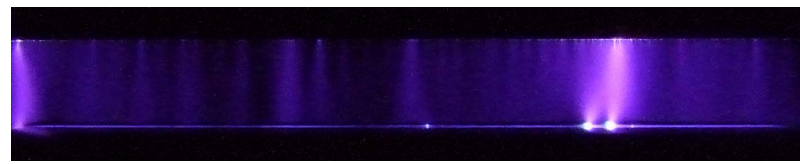

(b1)

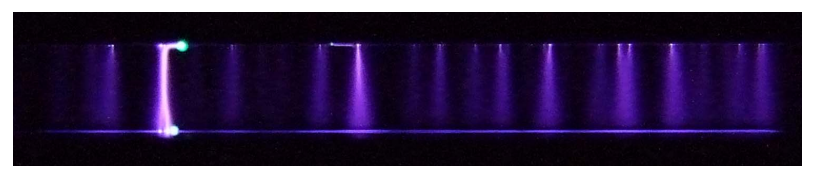

(b2)

Figure 2. Pulsed discharge images with different pulse risetimes at different air pressures ((a1) $20 \mathrm{~ns} / 6 \mathrm{kPa}$; (a2) 200 ns/6 kPa; (b1) 20 ns/15 kPa; (b2) 200 ns/15 kPa).

determined by the pulsed discharge time. Furthermore, due to the high resolution and the high sensitivity of the ordinary camera, detail of the discharge channel can be observed more clearly. Therefore, the images of the discharges were captured by a camera (FUJIFILM FinePix S6500) with a resolution of $2848 \times 2136$ pixels in a single shot with the exposure time of (1/3) s.

\section{Experimental Results and Discussions}

\subsection{Effect of Pulse Risetime}

Figure 2 shows the images of pulsed glow discharge in wire-plane air gap with the pulse risetime of $20 \mathrm{~ns}$ and

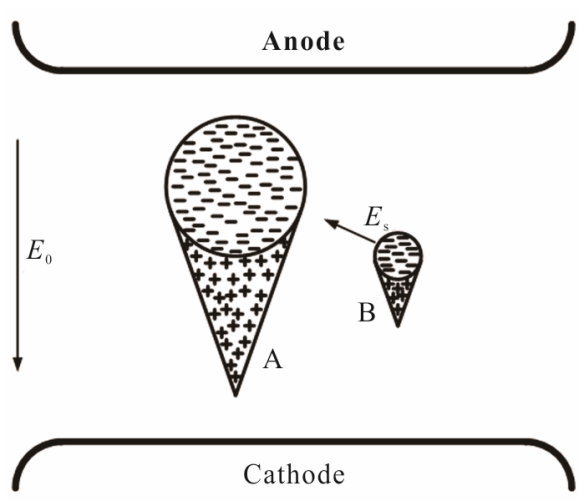

Figure 3. Schematic diagram of inter-shielding-effect between avalanches.

200 ns respectively. The pulse width and peak amplitude are fixed at $200 \mathrm{~ns}$ and $20 \mathrm{kV}$ respectively. Experiments were carried out at the air pressure of $6 \mathrm{kPa}$ and $15 \mathrm{kPa}$. For all images in this paper, the upper is wire anode electrode and the opposite is plane ground electrode.

From Figure 2, it can be seen that at the same gas pressure, in the case of fast pulse with risetime of $20 \mathrm{~ns}$, the white spots called spot glow by Anatoly Nikolaevich Maltsev near anode [14], are much more in number than those in the case of the slow pulse with risetime of 200 ns. This can be explained by the inter-shielding-effect. Because of the different delay times in the formation of the primary electrons around the anode, some avalanches grow ahead and distort the electric field in the gap, which leads to the suppressing of the adjacent avalanches. The schematic diagram of inter-shielding-effect is illustrated in Figure 3, in which $E_{0}$ is the applied electric field, $\mathrm{A}$ is the preceding avalanche, and $B$ is the posterior avalanche, $E_{\mathrm{S}}$ is the space charge electric field formed by A around the head of the posterior avalanche B. When $E_{\mathrm{S}}$ is large enough to cause the total electric field unsatisfied for the development of $\mathrm{B}$, the growth of the posterior avalanche B will stop.

Assuming that there is the delay time $\left(\tau_{0}\right)$ of the primary electron formation. Avalanche A grows ahead of avalanche $\mathrm{B}$ at the time of $\tau_{0}$ early. The growth of avalanche A results in accumulation of the space charges in the head of avalanche $A$ as well as the decrease of electric field $E_{\mathrm{S}}$ around the head of the posterior avalanche B, which leads to the suppressing of avalanche $B$ and the decrease of white spots around the anode. The following expression can also clarify the effect of pulse risetime on glow discharge [15].

$$
\frac{1}{2} n \mu\left(\frac{E}{n}\right)_{0} t_{0}<r_{c}, r_{c}=\left(40 \xi_{c} / n Q\right)^{1 / 2}
$$

where, $t_{0}$ is the pulse risetime when applied $E / n$ has reached $(E / n)_{0}, r_{c}$ is critical radius of avalanche head, $\mu$ is the electron mobility, $\xi_{c}$ is critical length of avalanche, $Q$ 
is the averaged momentum transfer cross section. From expression (1), we can see that with the decrease of pulse risetime, expression (1) can be quite easily satisfied, i.e. the overlapping condition between electron avalanches can be fulfilled. Furthermore, with the increase of air pressure, the risetime of pulse is required to decrease further to fulfill expression (1).

\subsection{Effect of Pulse Amplitude}

Figure 4 shows the images of the pulsed glow discharge under the pulse amplitude of $20 \mathrm{kV}$ and $35 \mathrm{kV}$ respectively. The experiments were carried out at different gas pressures: $20 \mathrm{kPa}, 40 \mathrm{kPa}$ and $60 \mathrm{kPa}$. The pulse risetime and pulse width are fixed at 20 ns and 120 ns respectively.

In Figure 4, the number of discrete channels in pulsed glow discharge decreases with the increase of peak amplitude. Moreover, the discrete channels can easily come

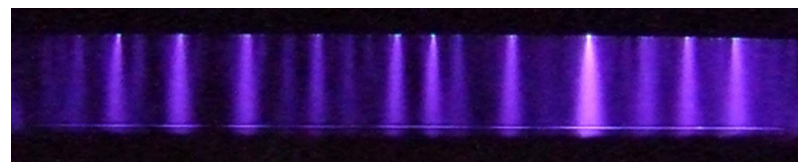

(a1)

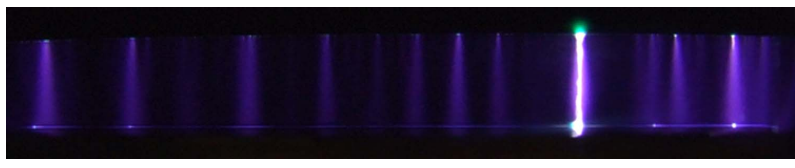

(a2)

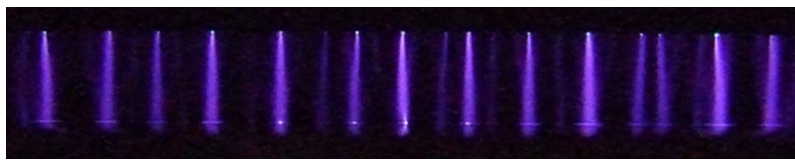

(b1)

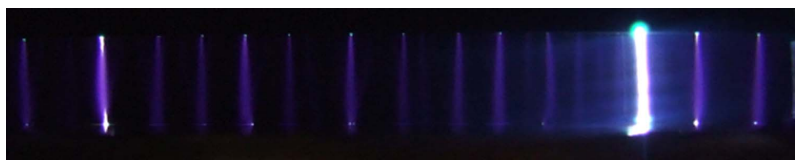

(b2)

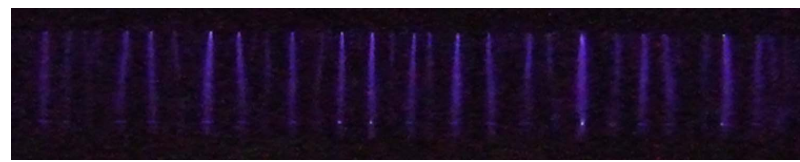

(c1)

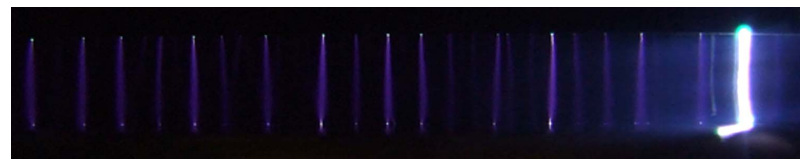

(c2)

Figure 4. Pulse discharge images with different pulse peak amplitude at different air pressure ((a1) $20 \mathrm{kPa} / 20 \mathrm{kV}$; (a2) $20 \mathrm{kPa} / 35 \mathrm{kV}$; (b1) $40 \mathrm{kPa} / 20 \mathrm{kV}$; (b2) $40 \mathrm{kPa} / 35 \mathrm{kV}$; (c1) $60 \mathrm{kPa} / 20 \mathrm{kV}$; (c2) $60 \mathrm{kPa} / 35 \mathrm{kV}$ ). into streamer discharge (the white channels illustrated in Figures 4(a2)-(c2) in the case of higher pulse amplitude due to the current heat effect. As mentioned above, the early or late growth of avalanches will form the uneven distribution of the current in each discrete channel. With the increase of the pulse amplitude, the higher overvoltage promotes the growth of avalanche $A$ as well as the formation of space charge electric field $\left(E_{S}\right)$, which results in the further increase of inter-shielding-effect on avalanche B. Therefore, the increase of pulse amplitude will cause the decrease of the discrete channel number as well as the increase of current in each channel. Due to the uneven distribution and the increase of the current in discrete channels, the early propagated channel will transit into streamer or spark discharge, which results in the current and the luminescence decrease in other discrete channels, as shown in Figures 4(a2)-(c2).

The discharge current waveforms with different pulse amplitude at the air pressure of $20 \mathrm{kPa}$ are illustrated in Figures 5(a) and (b). It can be found that there are some fluctuations on the current waveforms, which are related to the formation of avalanches during the discharge process. Due to the greater inhibition between avalanches at high pulse amplitude, the number of fluctuations on the current waveform under $35 \mathrm{kV}$ pulse (Figure 5(b)) is

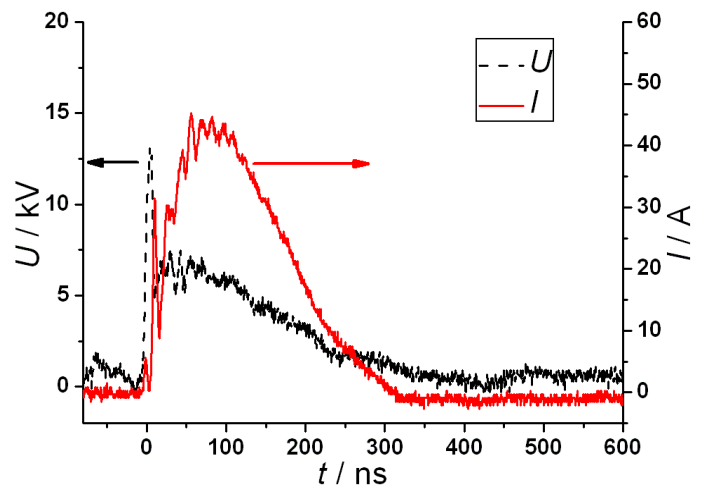

(a)

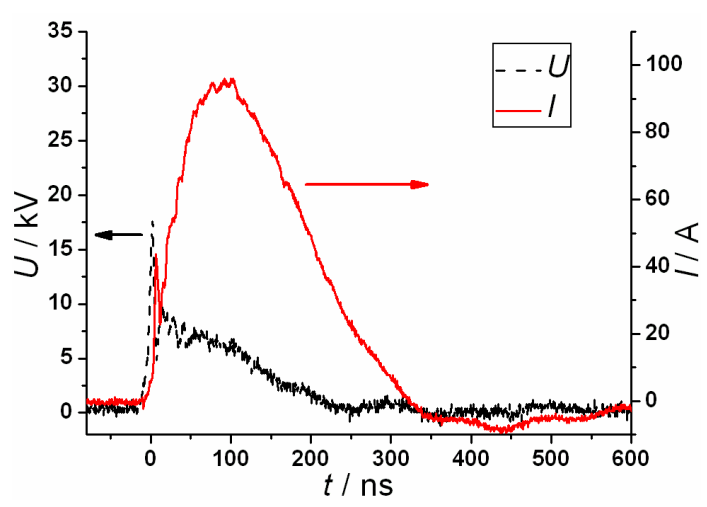

(b)

Figure 5. Current waveforms of pulse discharge under different amplitude in $20 \mathrm{kPa}$. (a) $U_{\mathrm{p}}=20 \mathrm{kV}$; (b) $U_{\mathrm{p}}=35 \mathrm{kV}$. 
less than that under $20 \mathrm{kV}$ pulse (Figure 5(a)).

From Figure 5, It can also be found that due to the streamer discharge, the peak discharge current under 35 $\mathrm{kV}$ pulse is larger than that under $20 \mathrm{kV}$ pulse, the voltage collapse time under $35 \mathrm{kV}$ pulse is less than that under $20 \mathrm{kV}$ pulse. A slight oscillation on the tail of the current waveform under the pulse amplitude of $35 \mathrm{kV}$ can also be seen, which is caused by the streamer discharge illustrated in Figure 4.

\subsection{Effect of Pulse Width}

Effect of the pulse width on the pulsed glow discharge is investigated by varying the pulse width from 120 ns to $1600 \mathrm{~ns}$ at the air pressure of $40 \mathrm{kPa}$. Figure 6 illustrates the images of large volume discharges under different pulse width with the fixed pulse risetime of $20 \mathrm{~ns}$ and peak amplitude of $20 \mathrm{kV}$.

From Figure 6, it can be clarified that the pulse width in the range from $120 \mathrm{~ns}$ to $1600 \mathrm{~ns}$ has little influence on the number and luminescence of the discrete channels in the pulsed glow discharge, but the increase of the pulse width will cause the transition from glow discharge to streamer or spark discharge easily. As discussed in part A, the discharge mode in the gap applied by pulsed voltage is dominated by the pulse risetime. Therefore, for a given pulse risetime, the number of the discrete channels is basically invariable and is not affected by the pulse width as shown in Figure 6. However, when increasing the pulse width, the higher energy will be injected into the discharge channel, which results in the

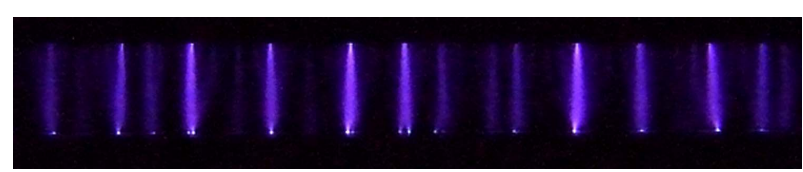

(a)

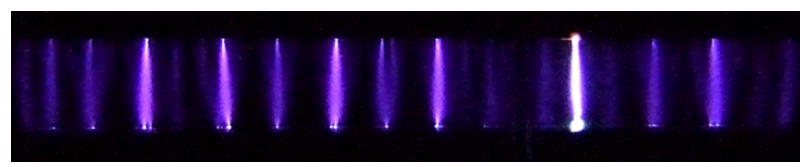

(b)

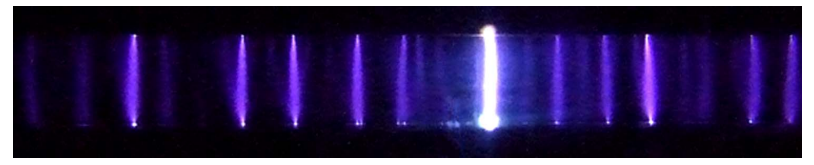

(c)

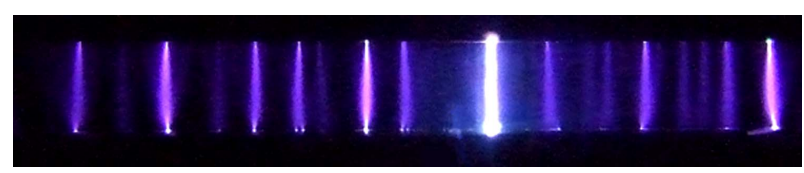

(d)

Figure 6. Images of pulse discharge with different pulse width (a) 120 ns; (b) 200 ns; (c) 500 ns; (d) 1600 ns. transition from glow discharge to streamer or spark discharge in the early propagated channel due to the uneven distribution of the current. In Figure 6, the luminescence of the other discrete channels is nearly not influenced by the pulse risetime, which can be considered that the increased pulse energy is almost injected into the early propagated channel with the increase of pulse width.

The discharge current waveforms under different pulse width are illustrated in Figure 7, the part in the range of 0 - 150 ns is zoomed at the top right corner. From Figure 7 , it can be seen that at the first $60 \mathrm{~ns}$, the pulse discharge current waveforms for different pulse width are almost the same, which is controlled by the growth of the pulsed glow discharge channels as shown in Figure 6, i.e. the number of the discrete channels as well as its distribution is determined by the pulse risetime. After the pulse reaches the peak value, the discharge development is determined by the pulse width. With the increase of the pulse width, the increased energy is injected into the early propagated channel, resulting in the increasing of the discharge current including the increase of the luminescence in the early propagated channel.

\section{Conclusion}

In this paper, pulsed glow discharge is generated in air between wire and plane electrodes with non-uniform electric field. The effect of pulse risetime, pulse width and amplitude on glow discharge has been widely investigated. Results reveal that a large volume glow discharge can be generated in an inhomogeneous electric field more easily under the pulse with faster risetime. The pulse width has almost no influence on the mode of glow discharge, but has a great influence on the transition from glow discharge to streamer discharge. A model of inter-shielding-effect has been proposed to explain the

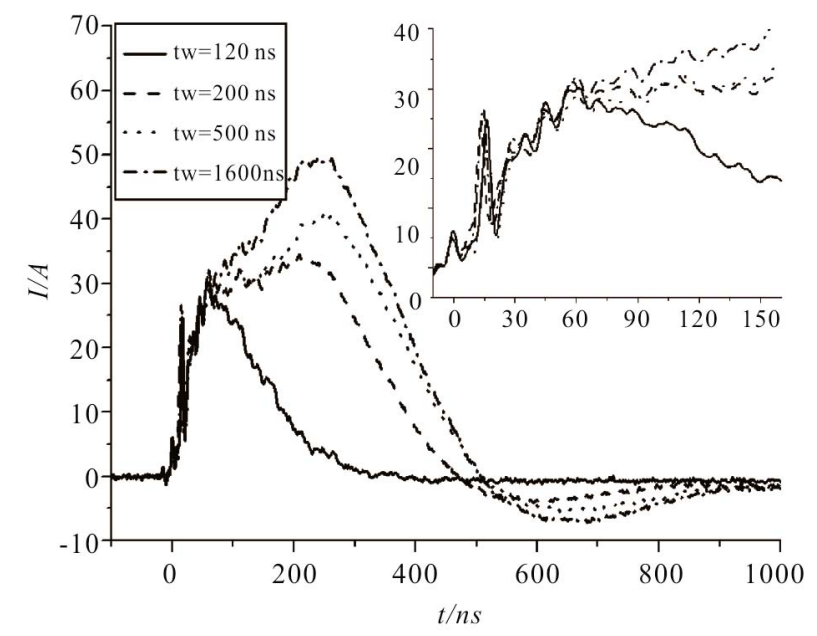

Figure 7. Pulse discharge current under different pulse width. 
influence of waveform parameters on the pulsed glow discharge.

\section{REFERENCES}

[1] W. Samaranayake, Y. Miyahara, T. Namihira, et al., "Ozone Generation in Dry Air Using Pulsed Discharges with and without a Solid Dielectric Layer," IEEE Transactions on Dielectrics and Electrical Insulation, Vol. 8, No. 4, 2001, pp. 687-697. doi:10.1109/94.946724

[2] R. Ono and T. Oda, "Ozone Production Process in Pulsed Positive Dielectric Barrier Discharge,” Journal of Physics D: Applied Physics, Vol. 40, No. 1, 2007, pp. 176-182. doi:10.1088/0022-3727/40/1/011

[3] M. Simek and M. Clupek, "Efficiency of Ozone Production by Pulsed Positive Corona Discharge in Synthetic Air,” Journal of Physics D: Applied Physics, Vol. 35, No. 11, 2002, pp. 1171-1175. doi:10.1088/0022-3727/35/11/311

[4] Y. Yankelevich and A. Pokryvailo, "High-Power ShortPulsed Corona: Investigation of Electrical Performance, $\mathrm{SO}_{2}$ Removal, and Ozone Generation," IEEE Transactions on Plasma Science, Vol. 30, No. 5, 2002, pp. 1975-1981. doi:10.1109/TPS.2002.805320

[5] F. Fresnet, G. Baravian, L. Magne, et al., "Influence of Water on NO Removal by Pulsed Discharge in $\mathrm{N}_{2} / \mathrm{H}_{2} \mathrm{O}$ / NO Mixtures,” Plasma Source Science and Technology, Vol. 11, No. 2, 2002, pp. 152-160. doi:10.1088/0963-0252/11/2/305

[6] T. Namihira, S. Tsukamoto, D. Wang, et al., "Influence of Gas Flow Rate and Reactor Length on NO Removal Using Pulsed Power," IEEE Transactions on Plasma Science, Vol. 29, No. 4, 2001, pp. 592-598. doi:10.1109/27.940952

[7] T. Yuji and Y. Sung, "Surface Treatment of $\mathrm{TiO}_{2}$ Films by Pulse Plasma for Dye-Sensitized Solar Cells Application,” IEEE Transactions on Plasma Science, Vol. 35, No.
4, 2007, pp. 1010-1013. doi:10.1109/TPS.2007.896918

[8] N. Takeuchi, K. Yasuoka and S. Ishii, "Surface Modification of Thin Rods by Theta-Pinching Metallic Plasmas," IEEE Transactions on Plasma Science, Vol. 34, No. 4, 2006, pp. 1112-1115. doi:10.1109/TPS.2006.876484

[9] W. D. Yang, P. N. Wang, F. M. Li and K. W. Cheah, "Synthesis of Oxygen-Free Nanosized InN by Pulse Discharge,” Nanotechnology, Vol. 13, No. 1, 2002, pp. 65-68. doi:10.1088/0957-4484/13/1/315

[10] H. Yang, W. Nam and D. Park, "Production of Nanosized Carbon Black from Hydrocarbon by a Thermal Plasma," Journal of Nanoscience and Nanotechnology, Vol. 7, No. 11, 2007, pp. 3744-3749. doi:10.1166/jnn.2007.003

[11] R. Ono and T. Oda, "Dynamics of Ozone and OH Radicals Generated by Pulsed Corona Discharge in Humid-Air Flow Reactor Measured by Laser Spectroscopy," Journal of Applied Physics, Vol. 93, No. 10, 2003, pp. 5876-5882. doi:10.1063/1.1567796

[12] R. Roush, R. Hutcherson, M. Ingram, et al., "Effects of Pulse Risetime and Pulse Width on the Destruction of Toluene and $\mathrm{NO}_{X}$ in a Coaxial Pulsed Corona Reactor," Proceeding of 22nd IEEE International Power Modulator Symposium, Boca Raton, 25-27 June 1996, pp. 79-84.

[13] D. Wang, M. Jikuya, S. Yoshida, et al., "Positive- and Negative-Pulsed Streamer Discharges Generated by a 100-ns Pulsed-Power in Atmospheric Air," IEEE Transacitions on Plasma Science, Vol. 35, No. 4, 2007, pp. 1098-1103. doi:10.1109/TPS.2007.902132

[14] A. N. Maltsev, "Dense Gas Discharge with Runaway Electrons as a New Plasma Source for Surface Modification and Treatment," IEEE Transactions on Plasma Science, Vol. 34, No. 4, 2006, pp. 1166-1174. doi:10.1109/TPS.2006.878994

[15] J. I. Levatter and S.-C. Lin, "Necessary Conditions for the Homogeneous Formation of Pulsed Avalanche Discharge at High Gas Pressure,” Journal of Applied Physics, Vol. 51, No. 1, 1980, pp. 210-222. doi:10.1063/1.327412 